\title{
Dapsone Syndrome With Acute Renal Failure During Leprosy Treatment: Case Report
}

\author{
Edson Nogueira Alves-Rodrigues, Luciano Correa Ribeiro, \\ Margareth Dióz Silva, Arley Takiuchi and \\ Cor Jesus Fernandes Fontes
}

\author{
Medical Clinics Department, Centre of Infectious \\ Diseases and Tropical Diseases Research of Mato Grosso, \\ Júlio Müller Hospital, Cuiabá, MT, Brazil
}

\begin{abstract}
Dapsone syndrome is a rare hypersensitivity reaction to dapsone and is characterized by high fever, papular or exfoliative dermatitis, progressing to liver toxicity and generalized lymphadenopathy, resembling a mononucleosis infection. We report a patient who developed acute renal failure, as well as other complications characteristic of dapsone syndrome, during leprosy treatment. Renal involvement had not been previously described as a dapsone syndrome feature.

Key Words: Leprosy, dapsone syndrome, acute renal failure.
\end{abstract}

The leprosy drugs, rifampicin and dapsone, can cause significant, unpredictable, skin, liver and hematological toxicity. A serious hypersensitivity reaction to dapsone has been described in $5 \%$ of the patients chronically using this drug, with onset two to six weeks after initiating therapy [1]. One of these reactions, called dapsone syndrome, is characterized by high fever, malaise, papular or exfoliative dermatitis, progressing to liver toxicity (jaundice, hepatitis and hepatomegaly), and generalized lymphadenopathy, resembling a mononucleosis infection. Fatalities are uncommon, but have been reported [1,2]. We report here a patient who developed acute renal failure, as well as complications similar to those seen in dapsone syndrome, during leprosy treatment.

\section{Case Report}

A 39-year-old female, recently diagnosed with indeterminate leprosy, was prescribed 600mg

Received on 22 October 2004; revised 10 January 2005.

Address for correspondence: Dr. Cor J F Fontes, Rua W Bras, 355 - M Sol, Zip code: 78043-508, Cuiabá, Mato Grosso, Brazil. Phone \#: 65 621-1406, Fax \#: 65 642-5149.

E-mail: fontes@terra.com.br

The Brazilian Journal of Infectious Diseases 2005;9(1):84-86 (C) 2005 by The Brazilian Journal of Infectious Diseases and Contexto Publishing. All rights reserved. rifampicin monthly and 100mg dapsone daily. After 23 days of therapy she stopped treatment because of a low fever, headache, dizziness and weakness. She denied previous allergies. Since her clinical condition worsened, she sought medical evaluation eight days after symptoms began. On presentation, in addition to the symptoms described above, the patient was toxic, with fever $\left(39.5^{\circ} \mathrm{C}\right)$, dry cough, respiratory discomfort, jaundice, hepatomegaly and hypotension $(\mathrm{Bp}=80 / 60 \mathrm{mmHg})$. Laboratory findings were as follows: hemoglobin $=11.2 \mathrm{~g} / \mathrm{dL}$; white blood cells $=12,700 / \mathrm{mm}^{3}$ (75\% granulocytes and $20 \%$ lymphocytes); first hour erythrocyte sedimentation rate $=77 \mathrm{~mm} ; A L T=137 \mathrm{U} / \mathrm{L} ; A S T=139 \mathrm{U} / \mathrm{L}$; unconjugated hyperbilirubinemia $=1.8 \mathrm{mg} / \mathrm{dL}$. The chest $\mathrm{x}$-ray was normal. Serological tests were negative for the following: hepatitis A, B and C, HIV, Epstein-Barr virus, leptospirosis, Trypanosoma cruzi, syphilis, typhoid fever, brucellosis, legionella, Yersinia enterocolitic (serotypes 3 and 9), toxoplasmosis $(\operatorname{IgM})$, rubella, cytomegalovirus ( $\operatorname{IgM})$, Mycoplasma pneumoniae (IgM). No malarial parasites were seen in the peripheral blood films. Antistreptolysin O, antinuclear antibodies, antiCrithidia luciliae DNA, anti-histone, anti-SS-A/Ro and SS-B/LA, antineutrophil cytoplasmic antibodies, and cryoglobulin serum tests were negative. 
After collecting blood and urine samples for microbiological cultures, wide spectrum antibiotic therapy (ceftriaxon and oxacilin) was initiated because of the patient's deteriorating condition. The patient then developed facial edema and pruritus, with a vesiculopustular skin rash. Lymph node enlargement developed in the cervical, axillary and inguinal regions. Fever persisted and the skin rash worsened to exfoliative erythroderma, associated with purpura on the lower extremities. The levels of serum-conjugated bilirubin increased to $7.8 \mathrm{mg} / \mathrm{dL}$. ALT and AST levels rose to 527 units/L and 236units/L, respectively. Subsequent laboratory investigations showed a lowering of hemoglobin levels, with a positive Coombs direct test. The white blood cell count increased to 29,060/ $\mathrm{mm}^{3}$, with eosinophilia (10.9\%). Serum immunoglobin E levels were high (1699Kunits/L) and C3/C4 complement levels were normal. Severe acute renal failure and oliguria developed. Urinalysis revealed hematuria and white cell casts, with no eosinophiluria. Hemodialysis was initiated. Kidney and skin biopsies were performed on the $7^{\text {th }}$ day after admission. Since it was assumed that the patient had dapsone syndrome, methylprednisolone (1,000 mg daily, for three days) and 1,000 mg cyclophosphamide (single dose) were started on the $8^{\text {th }}$ day of hospitalization, followed by $40 \mathrm{mg}$ prednisone daily, as maintenance therapy.

The patient's renal function and dermatological condition returned to normal two months after admission. Since fever and respiratory symptoms persisted, pulmonary tuberculosis was suspected and confirmed by sputum culture for Mycobacterium tuberculosis. Treatment was initiated with rifampicin, isoniazyd and pyrazinamide. The patient was followed for six months as an outpatient, receiving only tuberculosis therapy. At the end of six months the patient continued with rifampicin and clofazimin for leprosy, without further problems.

The kidney histopathological study revealed an interstitial vasculitis, with cellular infiltrates in arched and interlobular arteries, composed mainly of lymphocytes. Direct immunofluorescence of the glomeruli for IgA, IgD, IgE, IgG, IgM, C3, C1q and fibrin was negative. The skin biopsy showed dermal edema, extravasation of red blood cells, perivascular lymphocytic infiltration and edematous swelling of the endothelial cells, suggesting hypersensitivity vasculitis.

\section{Discussion}

Dapsone has been used for over 40 years for the treatment of a variety of conditions, and it is the most widely-used drug for leprosy treatment. This drug is well absorbed orally and is mainly eliminated in the urine. Dapsone can be found in the blood more than 35 days after treatment is suspended. Although it is considered a safe drug, several side effects have been reported. In general, adverse effects rarely occur at doses less than $100 \mathrm{mg}$ per day. They mainly consist of skin, nervous system, gastrointestinal tract, liver, kidney and hematological disorders. The most important are hemolytic anemia and methemoglobinemia. Hemolysis usually occurs at doses of up to $200 \mathrm{mg}$ daily, in patients with glucose-6-phosphate dehydrogenase deficiency [2]

This case illustrates classic dapsone syndrome, provoked by multi-drug therapy (MDT) for leprosy. The skin manifestations, lymphadenopathy and hepatitis fit the syndrome criteria [2]. Since MDT began to be used in leprosy patients, an increasing number of dapsone reactions have been reported throughout the world [3]. However, acute renal failure had not been previously described as a dapsone syndrome feature.

Although the pathogenesis of the dapsone syndrome is not well understood, findings of positive lymphocyte stimulation tests and predominantly activated cytotoxic $T$ cells in the dermis of patients with this syndrome suggest an allergic rather than an idiosyncratic reaction [4]. The skin rash and kidney lesions presented by our patient showed a hypersensitivity vasculitis histopathology, with lymphocyte infiltration and no hypocomplementemia [5]. The hypersensitivity vasculitis can be divided into neutrophylic and lymphocytic subtypes, corresponding to type III and type IV hypersensitivity immune reactions, respectively. The former is frequently associated with hypocomplementemia, whereas the latter is not [6]. Peripheral eosinophilia has been described [7]. 
Rifampicin, which is also used in MDT, is a strong inducer of microsomal enzymes, diminishing the pharmacological effects of a wide variety of drugs, including dapsone. Thus, any putative interaction of rifampicin predisposing to dapsone syndrome in our patient would not be a consequence of direct enhancement of dapsone toxicity [1].

Kidney involvement was unexpected. As dapsone is not associated with renal vasculitis, rifampicin toxicity might be considered an explanation of the kidney histopathology. However, rifampicin is most commonly cited as a drug causing tubulo-interstitial lesions, with eosinophilic and mononuclear cellular infiltration [8]. Furthermore, since the patient was subsequently treated with a six-month course of rifampicin for tuberculosis without any adverse reactions, the possibility of rifampicin-induced renal toxicity seems unlikely.

This patient exemplifies a serious dapsone reaction. Aggressive immune-suppressive therapy was used because of the severe renal failure; this therapy had a favorable outcome. Since dapsone is widely used for leprosy and other diseases, such as dermatitis herpetiformis, acne vulgaris, psoriasis, and Pneumocystis carinii pneumonia in acquired immunodeficiency syndrome, clinicians should be made aware of this life-threatening reaction.

\section{References}

1. Lau G. A fatal case of drug-induced multi-organ damage in a patient with Hansen's disease: dapsone syndrome or rifampicin toxicity? Forensic Sci Int 1995; 73:109-15.

2. Richardus J.H, Smith T.C. Increased incidence in leprosy of hypersensitivity reactions to dapsone after introduction of multidrug therapy. Lepr Rev 1989;60:267-73.

3. Reeve P.A., Ala J., Hall J.J. Dapsone syndrome in Vanuatu: a high incidence during multidrug therapy (MDT) of leprosy. J Trop Med Hyg 1992;95:266-70.

4. Saito S., Ikezawa Z., Miyamoto H., Kim S. A case of the 'dapsone syndrome'. Clin Exp Dermatol 1994;19:152-6.

5. Islek I., Baris S., Katranci A.O., et al. Hypersensitivity vasculitis induced by cefoperazone/sulbactam. Ann Clin Microbiol Antimicrob 2003;2:1.

6. Lie J.T. Illustrated histopathologic classification criteria for selected vasculitis syndromes. Arthr Rheum 1990;33:1074-87.
7. Fauci A.S. Predominantly cutaneous vasculitis. In: Braunwald E., Fauci A.S., Kasper D.L., et at., [eds.] Harrison's Principles of Internal Medicine. New York: McGraw-Hill $15^{\text {th }}$ edition, 2001.

8. Dedhia N.M., Almeida A.F., Khanna U.B., et al. Acute renal failure: a complication of new multidrug regimen for treatment of leprosy. Int J Lepr 1986;54:380-382. 